\title{
Retiming of short Pulses Using Quadratic Cascading in a Periodically Poled Lithium Niobate Waveguide
}

\author{
Sheng Liu, Kwang Jo Lee, Francesca Parmigiani, Katia Gallo, Periklis Petropoulos, \\ David J. Richardson
}

\begin{abstract}
We demonstrate an all-optical technique for the mitigation of timing jitter in short pulse transmission systems. The technique relies on pulse pre-shaping followed by optical switching in a periodically poled lithium niobate waveguide via cascaded second harmonic and difference frequency generation. The original data pulses are shaped into flat-top pulses to avoid conversion of their timing jitter into pulse amplitude noise at the output of the waveguide. Pulse retiming is confirmed by temporal measurements, while bit-error rate measurements confirm good noise performance for the system.
\end{abstract}

Index Terms - All-optical regeneration, optical pulse shaping, timing jitter, nonlinear optical process, harmonic generation and mixing, lithium niobate.

\section{INTRODUCTION}

$\mathrm{A}_{\mathrm{es}}^{\mathrm{LL}}$ LL-optical signal regeneration is expected to play an essential role in future high speed optical communication systems. The cumulative effects of repeater noise amplification and optical nonlinearities in long-haul transmission systems can give rise to both amplitude noise and timing jitter in the transmitted optical signals, resulting in significant performance degradation. Therefore, signal regeneration techniques (i.e. the mitigation of both amplitude noise and timing jitter) are required to overcome these transmission impairments.

Previous schemes for the mitigation of timing jitter from signals have relied on the generation of a temporal position-dependent frequency shift - imparted either by a synchronously driven phase modulator [1], or by cross-phase modulation in an optical fibre [2] - followed by a dispersive element to reposition the data pulse in the time domain. In different schemes the data pulses were pre-shaped into flat-top waveforms before being optically switched with clean,

Manuscript received June xx, 2010.

This work was supported by the UK EPSRC under grant agreement EP/F032218/1. K. Gallo gratefully acknowledges support from the EU under grant agreement PIEF-GA-2009-234798. S. Liu, K. J. Lee, F. Parmigiani, P. Petropoulos, and D. J. Richardson are with Optoelectronics Research Centre at University of Southampton, Southampton, United Kingdom (e-mail: shl@ orc.soton.ac.uk). K. Gallo is with the Department of Applied Physics, Royal Institute of Technology (KTH), 10691 Stockholm, Sweden.

Publisher Item Identifier synchronous pulses [3-5]. However, the speed of the scheme in [1] is limited by the electrical bandwidth of the modulator/drive

electronics rendering it unsuitable for high-speed optical time-division multiplexed signals, and the stability of the systems discussed in [2-4], which are based on $\chi^{(3)}$ nonlinearities in optical fibres, is generally compromised by the long fibre lengths required for efficient operation. Such limitations can be overcome using their compact $\chi^{(2)}$-based counterparts $[6,7]$.

In recent years, the use of cascaded second-order nonlinear processes in periodically poled lithium niobate (PPLN) waveguides has attracted considerable interest as a promising route to performing all-optical signal processing [8]. The technology provides for high nonlinear coefficients, low cross talk, an ultra-fast optical response, bit-rate and modulation format transparency, and no added spontaneous emission noise. Cascaded second-harmonic /difference-frequency generation (cSHG/DFG) and cascaded sum- and difference-frequency generation (cSFG/DFG) have both been exploited in various all-optical signal processing applications, such as wavelength conversion [9], format conversion [10], logic gates [11], tunable optical time delays [12], and phase sensitive amplification [13].

Previously, a retiming scheme that relied on the manipulation of the temporal walk-off between the pump and the second harmonic (SH) pulses in PPLN was presented in [7]. However, this scheme was limited in flexibility since the walk-off is directly related to the physical device length, and also the retiming region had an uneven intensity response which is likely to give rise to large amounts of amplitude noise on the output signal. In the experiment presented in this letter, we overcome these limitations by pre-shaping the data pulses into broader flat-top pulses, prior to them being launched into the waveguide, where they are switched with a synchronous clean optical clock $[6,14]$. We demonstrate the technique in a proof-of-principle experiment operating at $10 \mathrm{Gbit} / \mathrm{s}$ (with $7 \mathrm{ps}$ clock pulses), however much higher data rates can be achieved. The ultimate bandwidth limitation of our technique is imposed by the walk-off between the data and the SH signal. We estimate that operation at data rates up to $100 \mathrm{Gbit} / \mathrm{s}$ should be possible with the 30-mm PPLN devices used in our experiments. 


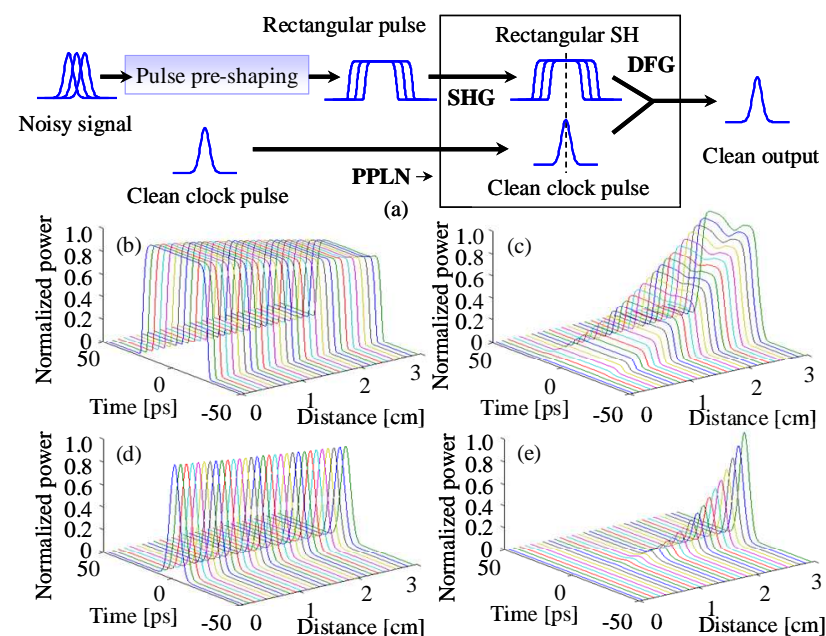

Fig. 1. (a) Illustration of the all-optical pulse retiming scheme based on both pulse pre-shaping and cSHG/DFG in a PPLN waveguide. Propagation of (b) input rectangular, (c) SH, (d) clean clock, and (e) output pulses along the length of the PPLN waveguide.

\section{OPERATION PRINCIPLE}

The basic idea of our retiming scheme is illustrated in Fig. 1(a). The input data pulses with large timing jitter are first pre-shaped into flat-top pulses, which in turn generate flat-top $\mathrm{SH}$ pulses by SHG in a PPLN waveguide. The SH pulses are then gated by the locally generated clock pulse train, which is synchronously launched into the PPLN device and temporally aligned to the nominal centre of the data pulses, to generate an output signal with suppressed timing jitter at a different wavelength. Note that clock recovery is normally required to synchronize the clock pulses to the incoming data stream.

Plots (b-e) in Fig. 1 show simulations of the propagation of initial flat-top, $\mathrm{SH}$, clock, and output pulses along the length of a 30-mm-long PPLN waveguide. The simulations used the coupled mode equations describing cSHG/DFG as reported in [10], with the same parameters as in the experiment described in the following section. As can be seen in Fig. 1(b) and (c), the initial flat-top pulse also generates a SH pulse with a flat-top shape and without any significant waveform distortion originating from walk-off effects in the device. The small dip on the flat-top region in the SH pulse is caused by the DFG interaction between the $\mathrm{SH}$ pulse and the clock pulse, and its position corresponds to the region of temporal overlap between them. The output pulse has a Gaussian profile, similar to the clock pulse, and its peak power grows gradually along the waveguide as shown in Fig. 1 (e).

\section{EXPERIMENT AND DISCUSSION}

Fig. 2 illustrates the experimental setup used to realise our all-optical pulse retiming system. A 30-mm-long fibre pigtailed PPLN waveguide (HC Photonics Corp., a z-cut proton-exchange PPLN waveguide) was used for the cSHG/ DFG process. Its SHG phase matching wavelength was 1546 $\mathrm{nm}$ at $50^{\circ} \mathrm{C}$, its insertion loss was $3.5 \mathrm{~dB}$ and its normalised (internal) conversion efficiency was $75 \% \mathrm{~W}^{-1} \mathrm{~cm}^{-2}$. An erbium glass oscillator was used as the pulse source for the data signal which comprised $10 \mathrm{GHz}, 7-p s$ pulses at $1546 \mathrm{~nm}$. The data pulses were amplitude modulated by a $2^{31}-1$ pseudorandom bit sequence (PRBS) using a lithium niobate modulator, then amplified and launched into an optical processor (Finisar WaveShaper 4000E) - a device that allows individual control over the intensity and phase of spectral components with a resolution of $4 \mathrm{GHz}$, - to form 50-ps-long (FWHM) rectangular pulses using Fourier shaping (Fig. 2(a) - details of the shaping technique can be found e.g. in [3]). The pulses had sharp trailing and leading edges and a good flat-top section. The clean clock pulses at a wavelength of $1550 \mathrm{~nm}$ were generated by a 10-GHz, 7-ps gain-switched laser (GSL) [see Fig. 2(b)], and amplified before being combined with the flat-top pulses using a $3-\mathrm{dB}$ coupler and launched into the PPLN waveguide. A variable delay line was used to control the relative position between the shaped data and the clean clock pulse trains and to simulate the effects of timing jitter. Polarization controllers were used to align all the optical waves to the optical axis of the PPLN waveguide. The average optical powers of both the data signal and the clock at the PPLN input were $18 \mathrm{dBm}$, and the optical signal to noise ratio (OSNR) of the data signal was $33 \mathrm{~dB}$. The (noisy) input reshaped pulses at $1546 \mathrm{~nm}$ were up-converted to $773 \mathrm{~nm}$ by the SHG process in the PPLN waveguide. The SH data stream, which would still have retained any timing jitter present on the data, was then gated by the input clock via DFG to mitigate the jitter and produce a clean replica of the original data at $1542 \mathrm{~nm}$. Figure 2(c) shows the spectrum at the output of the PPLN waveguide, showing that the output signal had an OSNR of more than $22 \mathrm{~dB}$. A tunable filter (Alnair Labs.) with a flat-top passband of $2 \mathrm{~nm}$ was used after the PPLN device to extract the retimed signal at $1542 \mathrm{~nm}$ and the corresponding measured eye-diagrams are shown in Fig. 2(d).

To assess the retiming performance of the system we varied systematically the relative delay between the clock and data signals and measured the output (Fig.3). In the figure, the temporal displacement $\Delta \tau=0$ corresponds to the clock pulse aligned to the nominal centre of the flat-top pulse. The
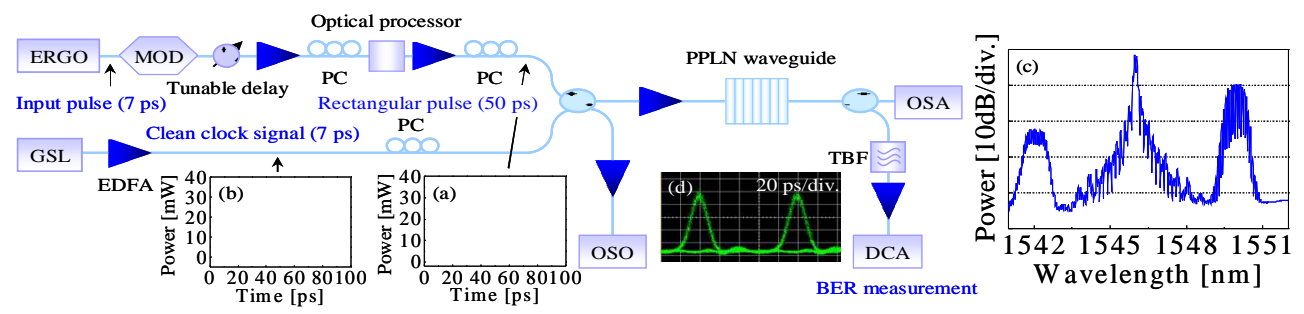

Fig. 2. Schematic of the experimental setup and experimental traces recorded on several points in the system: ERGO: erbium glass oscillator laser, GSL: gain switched laser, MOD: modulator, EDFA: erbium-doped fibre amplifier, PC: polarization controller, DCA: digital communication analyzer, OSO: optical sampling oscilloscope, TBF: tunable bandpass filter, OSA: optical spectrum analyser (measured with a resolution of $0.05 \mathrm{~nm}$ ). 


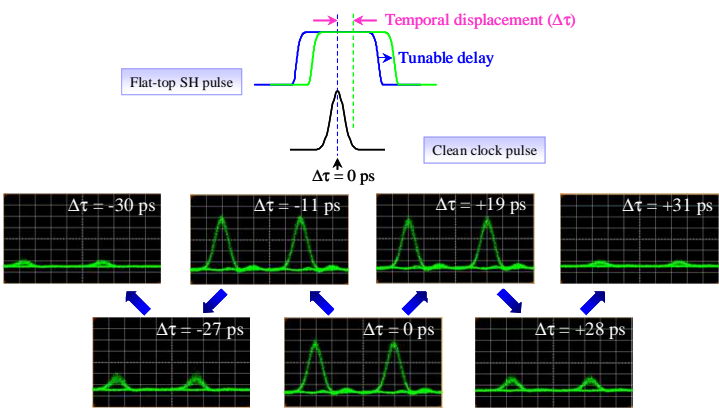

Fig. 3. Eye diagrams of the signal after retiming at various relative positions.
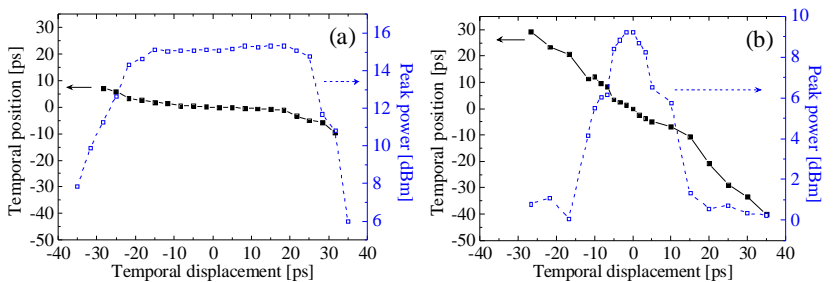

Fig. 4. Temporal position and peak power of the output signal plotted as a function of the input time delay (a) with and (b) without pre-shaping. temporal position of the output pulse did not change with the temporal offset between input and clock pulses, which means that any timing jitter on the input data pulses would not be transferred to the converted pulses. As long as the clock pulses overlapped with the flat-top regions of the $\mathrm{SH}$ pulses (50 ps), the peak powers of the output pulses remained constant as shown in Fig. 3. However, when the clock pulses moved away from the flat-top regions of the shaped pulses, the output peak powers dramatically decreased. This result indicates that the maximum tolerable input timing jitter is determined by the duration of the flat-top pulses.

The measured peak power and temporal position of the output pulses are plotted in Fig. 4 as a function of the temporal offset between the pulse streams. The zero on the horizontal axis was chosen to coincide with the point providing the most efficient conversion (or the maximum peak power). Fig. 4(a) corresponds to the case in which the input data pulses have been pre-shaped into flat-top pulses, and shows clearly the retiming performance of the device. In contrast, Fig. 4(b) corresponds to the case when no pulse shaping was applied to the input signal. This latter case shows very limited retiming capability because the pulse width of the unshaped SH pulses is comparable to that of the clock pulses [6].

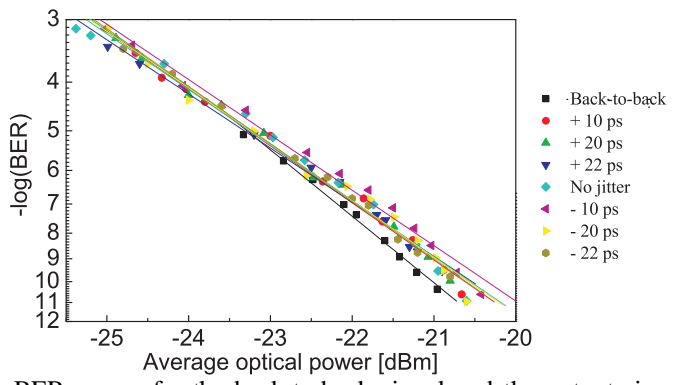

Fig. 5. BER curves for the back-to-back signal and the output signals with various time delays.

We finally performed bit-error rate (BER) measurements at $10 \mathrm{Gbit} / \mathrm{s}$ (using an electrically amplified receiver) to ensure that the retiming system did not introduce any amplitude noise on the input pulses (Fig. 5). Retiming of the output signal was realised with a slight power penalty of $0.6 \mathrm{~dB}$ (at $\left.\mathrm{BER}=10^{-9}\right)$ as compared to the back-to-back measurements. This penalty is mainly due to the OSNR degradation from $33 \mathrm{~dB}$ in the data signal to $22 \mathrm{~dB}$ in the switched output.

\section{CONCLUSION}

We have demonstrated an all-optical signal retiming system based on pulse shaping and cSHG/DFG-based switching in a fully fiberised 30-mm-long PPLN waveguide. In our current retiming scheme, the data signal has to be at the SHG phase matching wavelength of the PPLN device. This requirement could be overcome by employing the cSFG/DFG process instead, in which the data signal would act as one of the pumps for the SFG process. Our results have shown the benefits of pre-shaping the data pulses into a flat-top waveform, as opposed to feeding them directly into the PPLN waveguide. Error-free operation at $10 \mathrm{Gbit} / \mathrm{s}$ was obtained for various delays with a power penalty below $0.6 \mathrm{~dB}$. We believe that the technique could be extended directly to repetition rates of $\sim 100$ Gbit/s for a 30-mm-long device, however even higher repetition rates could be achieved in a shorter device.

\section{REFERENCES}

[1] L. A. Jiang, M. E. Grein, H. A. Haus, and E. P. Ippen, "Timing jitter eater for optical pulse trains," Opt. Lett. vol. 28, pp. 78-80, 2003.

[2] F. Parmigiani, P. Petropoulos, M. Ibsen, D. J. Richardson, "Pulse retiming based on XPM using parabolic pulses formed in a fiber Bragg grating", IEEE Photon. Technol. Let. vol.18, pp.829-831, 2006.

[3] F. Parmigiani, P. Petropoulos, M. Ibsen, and D. J. Richardson, "All-optical pulse reshaping and retiming systems incorporating pulse shaping fibre Bragg grating," J. Lightwave Technol. vol. 24, pp. 357-364, 2006.

[4] F. Parmigiani, L. K. Oxenløwe, M. Galili, M. Ibsen, D. Zibar, P. Petropoulos, D. J. Richardson, A. T. Clausen, and P. Jeppesen, “All-optical 160-Gbit/s retiming system using fibre grating based pulse shaping technology," J. Lightwave Technol. vol. 27, pp. 1135-1140, 2009.

[5] S. Watanabe, F. Futami, R. Okabe, Y. Takita, S. Ferber, R. Ludwig, C. Schubert, C. Schmidt-Langhorst and H. G. Weber, "160 Gb/s Optical 3R-Regenerator in Fiber Transmission experiment," in Proc. of OFC 2003, Paper PD16-1, 2003.

[6] K. Gallo, J. Prawiharjo, F. Parmigiani, P. Almeida, P. Petropoulos, and D. Richardson, "Processing ultrafast optical signals in broadband telecom systems by means of cascaded quadratic nonlinearities," presented at the Proc. ICTON, Nottingham, UK, 2006, Invited paper.

[7] T. Hasegawa, X. Wang, and A. Suzuki, "Retiming of picosecond pulses by a cascaded second-order nonlinear process in quasi-phasematched $\mathrm{LiNbO}_{3}$ waveguides," Opt. Lett. vol. 29, pp. 2776-2778, 2004.

[8] C. Langrock, S. Kumar, J. E. McGeehan, A. E. Willner, and M. M. Fejer, "All-optical signal processing using $\chi^{(2)}$ nonlinearities in guidedwave devices,” J. Lightwave Technol. vol. 24, pp. 2579-2592, 2006.

[9] K. Gallo, G. Assanto, and G. I. Stegeman, "Efficient wavelength shifting over the erbium amplifier bandwidth via cascaded second order processes in lithium niobate waveguides," Appl. Phys. Lett. vol. 71, pp. 1020-1022 1997.

[10] K. J. Lee, S. Liu, F. Parmigiani, M. Ibsen, P. Petropoulos, K. Gallo, and D. J Richardson, "OTDM to WDM format conversion based on quadratic cascading in a periodically poled lithium niobate waveguide," Opt. Express vol. 18, pp. 10282-10288, 2010.

[11] J. E. McGeehan, M. Giltrelli, and A. E. Willner, "All-optical digital 3-input AND gate using sum- and difference-frequency generation in a PPLN waveguide," Electron. Lett. vol. 43, pp. 409-410, 2007.

[12] Y. Wang, C. Yu, L. Yan, A. E. Willner, R. Roussev, C. Langrock, M. M. Fejer, J. E. Sharping, and A. L. Gaeta, "44-ns continuously tunable dispersionless optical delay element using a PPLN waveguide with two-pump configuration, DCF, and a dispersion compensator," IEEE Photon. Technol. Lett. vol. 19, pp. 861-863, 2007. 
[13] K. J. Lee, F. Parmigiani, S. Liu, J. Kakande, P. Petropoulos, K. Gallo, and D. Richardson, "Phase sensitive amplification based on quadratic cascading in a periodically poled lithium niobate waveguide," Opt. Express vol. 17, pp. 20393-20400, 2009.

[14] K. J. Lee, S. Liu, F. Parmigiani, P. Petropoulos, D. J. Richardson, "All-optical pulse retiming based on quadratic cascading in a periodically poled lithium niobate waveguide," presented at the Proc. Nonlinear Photonics, Karlsruhe-Messe und Kongress, Karlsruhe, Germany, 2010, Paper NMB5. 